\title{
Gadolinium-Based MRI Contrast Agents Induce Mitochondrial Toxicity and Cell Death in Human Neurons, and Toxicity Increases With Reduced Kinetic Stability of the Agent
}

\author{
Danielle V. Bower, MD, PhD, *广 Johannes K. Richter, MD, * Hendrik von Tengg-Kobligk, MD, *广 \\ Johannes T. Heverhagen, $M D, P h D$, *广 and Val M. Runge, $M D^{* \dagger}$
}

\begin{abstract}
Objectives: This preclinical study was devised to investigate potential cellular toxicity in human neurons induced by gadolinium-based contrast agents (GBCAs) used for contrast-enhanced magnetic resonance imaging (MRI). Neurons modeling a subset of those in the basal ganglia were tested, because the basal ganglia region is 1 of 2 brain regions that displays the greatest T1-dependent signal hyperintensity changes.

Methods: Eight GBCAs were tested. Dopaminergic neurons modeling a subset of those in the basal ganglia were differentiated from an established human neuroblastoma cell line and exposed to increasing concentrations of each agent for 7 days. The tested dosages ranged from clinically relevant concentrations measured in some autopsy patients who had received repeated injections of contrast for MRI, to higher concentrations to reveal dose-dependent toxicity trends. Cell death, mitochondrial membrane potential, mitochondrial oxidative capacity, and mitochondrial function measured by oxygen consumption were quantified in cells treated with each GBCA or the osmolality control mannitol and compared to untreated cells which served as a negative control.

Results: Mannitol caused no change from negative controls in any of the tests, at any concentration tested. For all GBCAs, cell death increased with exposure dose, with toxicity at clinically relevant doses for agents with lower kinetic stability. Reduction of mitochondrial membrane potential and oxidative respiratory function also generally mirrored the agents' structural kinetic stabilities, with greater impairment at lower concentration for the less stable agents.

Conclusions: In human neurons modeling a subset of those in the basal ganglia, these results demonstrate a toxic effect of gadolinium-containing MRI contrast agents on mitochondrial respiratory function and cell viability. Toxicity increases as agent concentration increases and as the kinetic stability of the agent decreases.
\end{abstract}

Key Words: contrast agent, toxicity, neuron, gadolinium,

magnetic resonance imaging

(Invest Radiol 2019;54: 453-463)

Received for publication January 8, 2019; and accepted for publication, after revision, March 1, 2019

From the *Department of Diagnostic, Interventional, and Pediatric Radiology, Inselspital, University Hospital of Bern; and $\dagger$ Department for BioMedical Research, University of Bern, Bern, Switzerland.

Johannes T. Heverhagen and Val M. Runge share the senior authorship.

Author contributions: D.V.B. and V.M.R. conceived of the overall project. D.V.B. conceived the experimental plans, designed the protocols, conducted the experiments, analyzed the data, and produced the final figures. J.K.R. validated reproducibility of quantifications as a second reader. D.V.B. wrote and revised the manuscript. H.v.T.K. and J.T.H. financed and oversaw the project. All authors discussed the results and approved the final manuscript.

This work was performed in the laboratory for Experimental Radiology, Inselspital, University Hospital of Bern, located in the Department for BioMedical Research (DBMR), University of Bern. Imaging was conducted at the Live Cell Imaging Core Facility of the Microscopy Imaging Center at the University of Bern. The experiments measuring oxygen consumption and acidification were performed in the laboratory of Stefan Freigang in the Institute of Pathology at the University of Bern.

This study was funded by the Department of Diagnostic, Interventional, and Pediatric Radiology, Inselspital, University Hospital of Bern, University of Bern, Bern, Switzerland.

The authors declare no conflicts of interest.

Correspondence to: Danielle V. Bower, MD, PhD, Department of Diagnostic, Interventional, and Pediatric Radiology, Inselspital, University Hospital of Bern, University of Bern, Freiburgstrasse 10, 3010 Bern, Switzerland. E-mail: danielle.bower@insel.ch. Copyright (C) 2019 Wolters Kluwer Health, Inc. All rights reserved.

ISSN: 0020-9996/19/5408-0453

DOI: $10.1097 /$ RLI.0000000000000567 adolinium-based contrast agents (GBCAs) for contrast-enhanced magnetic resonance imaging (MRI) have been used for decades and are generally well-tolerated and considered safe for use in patients who have normal kidney function. ${ }^{1}$ However, the known deposition of gadolinium in tissues throughout the body, and particularly, accumulation in regions of the brain, has renewed concerns about the long-term safety of these agents. Kanda et $\mathrm{al}^{2}$ first highlighted the changes in T1-dependent MRI signal intensity after MRIs without contrast agent in the brains of patients with normal renal function who had received multiple prior injections of gadolinium-containing contrast. The basal ganglia and cerebellar dentate nucleus are the brain regions that most strongly exhibit these effects.

Multiple subsequent studies have shown that such T1-dependent signal intensity changes persist in patients who have received repeated injections of the linear GBCAs, but repeated use of the macrocyclics results in minimal to no persistent change. ${ }^{3-12}$ To explain these findings, studies have evaluated the entry into and clearance of contrast agents from the brain. ${ }^{13-16}$ Experiments in rats provide evidence that the linear agents dissociate gadolinium from their chelate, which precipitates or binds to macromolecules and persists long-term within brain tissue. ${ }^{17}$ In contrast, the macrocyclic agents predominantly remain as soluble, intact complexes and are gradually removed from the brain within approximately 5 months. ${ }^{17-19}$ Increasing evidence argues that all agents reside within the interstitium of human brain tissue for some period of time, where they may be taken up into various cell types, including glia $^{20}$ and neurons. ${ }^{21}$

During this time, emerging reports of possible clinical symptoms including headache, confusion, and bone and limb pain experienced by patients after a contrast-enhanced MRI scan have been published. ${ }^{22-25}$ Recognition of these symptoms, the persistent T1-dependent signal intensity changes in the brain, and the detection of gadolinium in brain tissue of autopsy patients ${ }^{21,26,27}$ necessitate clarification as to whether these agents cause toxicity in the brain. Yet, to date, it remains unknown whether these agents induce toxic effects on the cellular function of human neurons.

In the current study, we sought to identify whether GBCAs are toxic to the cellular function of differentiated human neurons that are representative of those found in one of the brain regions with high T1-dependent MRI signal intensity changes. As such, we incubated a subset of basal ganglia neurons with concentrations of each contrast agent ranging from clinically relevant doses (based on reported amounts of gadolinium measured in some autopsy patients) to higher doses, and investigated dose-dependent effects on cell viability and function.

\section{MATERIALS AND METHODS}

\section{MRI Contrast Agents}

The commercial MRI contrast agents were obtained from the manufacturers as noted in Table 1. We were unable to obtain gadoversetamide, and we were only able to obtain gadopentetate dimeglumine 
TABLE 1. Contrast Agents

\begin{tabular}{|c|c|c|c|c|c|c|c|}
\hline $\begin{array}{l}\text { Generic Name } \\
\text { (Trade Name) } \\
\end{array}$ & Manufacturer & Structure & Ionicity & $\begin{array}{c}\text { Osmolality at } 37^{\circ} \mathrm{C} \\
\left(\mathrm{mOsm} / \mathrm{kg} \mathrm{H} \mathrm{H}_{2} \mathrm{O}\right) \dagger\end{array}$ & Excess Chelate & $\log K_{\text {cond }}+$ & $\begin{array}{c}\text { Dissociation Half-Life } \\
\mathrm{T}_{1 / 2} \text { (Kinetic Stability), } \\
\text { pH 1.0, } 25^{\circ} \mathrm{C} \\
\end{array}$ \\
\hline NF-gadodiamide & & Linear & Nonionic & 739 & 0 & 14.9 & $<5 \mathrm{~s}$ \\
\hline Gadodiamide (Omniscan) & GE Healthcare & Linear & Nonionic & 789 & $\begin{array}{l}25 \mathrm{mmol} / \mathrm{L} \text { caldiamide } \\
\mathrm{Na}+\text { salt } \\
\mathrm{Ca}-\mathrm{DTPA}-\mathrm{BMA}\end{array}$ & 14.9 & $<5 \mathrm{~s}$ \\
\hline $\begin{array}{l}\text { Gadopentetate dimeglumine } \\
\text { (Magnevision, Magnevist)* }\end{array}$ & $\begin{array}{l}\text { Bayer } \\
\text { Healthcare }\end{array}$ & Linear & Ionic & 1960 & $1 \mathrm{mmol} / \mathrm{L}$ free DTPA & 17.7 & $<5 \mathrm{~s}$ \\
\hline $\begin{array}{l}\text { Gadoxetate disodium } \\
\text { (Primovist/Eovist) }\end{array}$ & $\begin{array}{l}\text { Bayer } \\
\text { Healthcare }\end{array}$ & Linear & Ionic & 688 & $\begin{array}{l}1.5 \mathrm{mmol} / \mathrm{L} \text { trisodium } \\
\text { salt } \mathrm{Ca}-\mathrm{EOB}-\mathrm{DTPA}\end{array}$ & 18.7 & $<5 \mathrm{~s}$ \\
\hline $\begin{array}{l}\text { Gadobenate dimeglumine } \\
\text { (MultiHance) }\end{array}$ & $\begin{array}{l}\text { Bracco } \\
\text { Imaging }\end{array}$ & Linear & Ionic & 1970 & 0 & 18.4 & $<5 \mathrm{~s}$ \\
\hline $\begin{array}{l}\text { Gadoterate meglumine } \\
\text { (Dotarem) }\end{array}$ & Guerbet & Macrocyclic & Ionic & 1350 & 0 & 19.3 & $338 \mathrm{~h}$ \\
\hline $\begin{array}{l}\text { Gadobutrol } \\
\text { (Gadovist/Gadavist) }\end{array}$ & $\begin{array}{l}\text { Bayer } \\
\text { Healthcare }\end{array}$ & Macrocyclic & Nonionic & 1603 & $\begin{array}{l}1.0 \mathrm{mmol} / \mathrm{L} \mathrm{Na}+\text { salt } \\
\text { Ca-BT-DO3A }\end{array}$ & 14.7 & $43 \mathrm{~h}$ \\
\hline Gadoteridol (ProHance) & $\begin{array}{l}\text { Bracco } \\
\text { Imaging }\end{array}$ & Macrocyclic & Nonionic & 630 & $\begin{array}{c}0.5 \mathrm{mmol} / \mathrm{L} \mathrm{Ca}_{2}+\text { salt } \\
{[\mathrm{Ca}-\mathrm{HP}-\mathrm{DO} 3 \mathrm{~A}] 2}\end{array}$ & 17.1 & $3.9 \mathrm{~h}$ \\
\hline
\end{tabular}

*We were unable to obtain Magnevist, so we used Magnevision, which is a generic preparation with the same chemical formula as Magnevist.

$\dagger$ Osmolality of the pharmaceutical preparation, except for nonformulated gadodiamide, which is a nonpharmaceutical research preparation.

$\$$ Conditional thermodynamic stability constant at $\mathrm{pH} 7.4$ reflecting the affinity of gadolinium for its ligand.

The values in the table were obtained from Idée et al, ${ }^{28}$ Port et al, ${ }^{29}$ and Runge et al. ${ }^{30}$

as Magnevision rather than Magnevist, but these two have the same chemical formula. The noncomplexed gadodiamide was kindly provided by the research and development division of Guerbet. The values in the table were obtained from Idée et $\mathrm{al}^{28}{ }^{28}$ Port et $\mathrm{al}^{29}$ and Runge et al. ${ }^{30}$

\section{Cell Culture and Differentiation of \\ Dopaminergic Neurons}

SH-SY5Y human neuroblastoma cells were obtained from ATCC (CRL-2266, Lot \#63290668). Adherent and suspension cells were propagated at $37^{\circ} \mathrm{C}$ and $5 \% \mathrm{CO}_{2}$ in sterile filtered (ThermoFisher 450-0045) SH-SY5Y media (see Table 2 for all medias used), and passaged 1:10 or higher using Accutase (ThermoFisher A1110501) around $80 \%$ confluency. Cells were frozen in liquid nitrogen in SH-SY5Y

TABLE 2. Cell Culture Medias

\begin{tabular}{ll}
\hline SH-SY5Y Media & \multicolumn{1}{c}{ Differentiation Media 1 } \\
\hline $\begin{array}{l}\text { DMEM/F12 (Bioswisstec AG FG4815) } \\
\text { 15\% FBS (ThermoFisher 10270, }\end{array}$ & $1 \%$ FBS \\
$\begin{array}{l}\text { heat inactivated) } \\
\text { 1\% nonessential amino acids }\end{array}$ & $1 \%$ NEAA \\
(NEAA) (ThermoFisher 11140035) & \\
1\% penicillin/streptomycin (P/S) & $1 \%$ P/S \\
(ThermoFisher 15140122) & $10 \mu$ retinoic \\
& acid (Sigma R2625) \\
\hline Differentiation Media 2 & Phosphate-Free Incubation Media \\
\hline Neurobasal media (ThermoFisher & DMEM without phosphate \\
12348017) & (ThermoFisher 11971025) \\
1X B27 (ThermoFisher 17504044) & $1 \mathrm{X} \mathrm{B27}$ \\
$1 \%$ Glutamax (ThermoFisher 35050061) & $1 \%$ Glutamax \\
20 mM KCl & $20 \mathrm{mM} \mathrm{KCl}$ \\
$1 \%$ P/S & $1 \%$ P/S \\
$80 \mathrm{nM}$ phorbol 12-myristate 13-acetate & $80 \mathrm{nM} \mathrm{PMA}$ \\
(PMA) (Sigma P8139) & \\
\hline
\end{tabular}

media with 5\% vol/vol DMSO. To differentiate, $2-4 \times 10^{4} \mathrm{SH}-\mathrm{SY} 5 \mathrm{Y}$ cells were plated per well in $200 \mu \mathrm{L}$ media in 96-well imaging dishes (Eppendorf 0030 741.013). The following day, the media was changed to differentiation media 1 for 4 days, followed by a further 7 days in differentiation media 2 .

\section{Incubation of Neurons With MRI Contrast Agents and Assessment of Cellular Toxicity}

Following the 11-day differentiation protocol, neurons were cultured in phosphate-free media to avoid gadolinium phosphate precipitation upon addition of MRI contrast agents. Indeed, we did not observe visible precipitates in our cultures. Briefly, each contrast agent was diluted in water for injection to a stock concentration of $0.25 \mathrm{M}$. Each agent was then diluted sequentially to final concentrations of $10 \mathrm{mM}$, $1 \mathrm{mM}, 100 \mu \mathrm{M}$, and $50 \mu \mathrm{M}$ in phosphate-free media. Individual wells of neurons were incubated with each concentration of contrast agent in triplicate or more for 7 days.

After incubation with contrast agent, cellular toxicity was assessed by preincubating cells for 45 minutes with fluorescent indicators and imaging with a Zeiss LSM 710 confocal microscope and Plan-Apochromat $20 \times / 0.8$ M27 objective. For quantification of cell death, all cell nuclei were labeled with $5 \mu \mathrm{g} / \mathrm{mL}$ Hoechst 33342 (Thermo Scientific 62249), which is a classic compound used to stain DNA. ${ }^{31}$ The Hoechst 33342 was excited at $405 \mathrm{~nm}$ with emissions collected at 422 to $498 \mathrm{~nm}$ with a $4 \mu \mathrm{M}$ pinhole. All dead cells were labeled with $2 \mu \mathrm{g} / \mathrm{mL}$ propidium iodide (Sigma P4170), excited at $405 \mathrm{~nm}$ and imaged at 600 to $755 \mathrm{~nm}$. Apoptotic cells with active caspase 3 or 7 were labeled with $2 \mu \mathrm{M}$ CellEvent caspase 3/7 reagent (ThermoFisher C10723), excited at $488 \mathrm{~nm}$ and imaged at 492 to $568 \mathrm{~nm}$. Mitochondrial membrane potential was assayed with freshly prepared $10 \mu \mathrm{M}$ TMRE (Sigma 87917), excited at $561 \mathrm{~nm}$ and imaged at 570 to $754 \mathrm{~nm}$ with a $2 \mu \mathrm{m}$ pinhole and $2 \mu \mathrm{m}$ interval stack centered at the brightest plane. Mitochondrial oxidative capacity was assayed with freshly prepared $5 \mu \mathrm{M}$ DCFH2-DA (Sigma D6883), excited at $488 \mathrm{~nm}$ and imaged at 493 to $575 \mathrm{~nm}$ with a $2 \mu \mathrm{m}$ pinhole and $2 \mu \mathrm{m}$ interval stack centered at the brightest plane. Scan speed was 8 , with averaging of 2 and zoom 
1. When signal intensity was to be quantified, laser power and PMT gain were identical across all samples within an experiment, and normalization to negative controls lacking contrast agent was performed within each experiment.

Mannitol (Sigma M4125) was selected as an osmolality control. However, due to a miscalculation, the highest concentration of mannitol tested did not equal the osmolality of the high osmolality contrast agents when applied at the concentration of $10 \mathrm{mM}$. At $10 \mathrm{mM}$, the osmolality of the following agents are: gadopentetate dimeglumine, $39.2 \mathrm{mOsm} / \mathrm{kg}$; gadobenate dimeglumine, $39.4 \mathrm{mOsm} / \mathrm{kg}$; gadoterate meglumine, $27 \mathrm{mOsm} / \mathrm{kg}$; and gadoxetate disodium, $27.5 \mathrm{mOsm} / \mathrm{kg}$. The highest concentration of mannitol tested had an osmolality of $24 \mathrm{mOsm} / \mathrm{kg}$. All other contrast agents have an osmolality less than $24 \mathrm{mOsm} / \mathrm{kg}$ at a concentration of $10 \mathrm{mM}$, and their osmolalities were therefore properly controlled. At a concentration of $1 \mathrm{mM}$, the osmolality of all contrast agents is below $24 \mathrm{mOsm} / \mathrm{kg}$, and thus osmolality at this concentration was properly controlled.

Cumulative signal intensity summing all pixels in a region of interest was quantified for mitochondrial membrane potential and oxidative capacity using ImageJ with a $4.98 \times 4.98-\mu \mathrm{m}$ oval region of interest on the brightest image slice and averaging 15 or more cells per sample. To quantify cell death, all cells labeled by propidium iodide or activated caspase were individually counted and compared with the total number of nuclei (by Hoechst 33342 label) in the image. Repeat quantification reproducibility was within $1 \%$. Interreader reproducibility was within $2 \%$.

\section{Characterization of mRNA Expression}

mRNA was collected from SH-SY5Y neuroblasts and from neurons after differentiation plus an additional 7 days' culture in phosphatefree media using the Dynabeads mRNA Direct Kit (Ambion 61011). quantitative real-time polymerase chain reaction (qRT-PCR) was performed using the Luna Universal One-Step RT-qPCR Kit (New England BioLabs E3005) on an Applied Biosystems 7500 Real-Time Fast PCR machine. See Table 3 for the primers used. Results were analyzed using the $\Delta \Delta \mathrm{CT}$ method.

\section{Seahorse XFe Assessment of Mitochondrial Respiration}

Approximately $1 \times 10^{4} \mathrm{SH}-\mathrm{SY} 5 \mathrm{Y}$ cells were plated per well in Seahorse 96-well culture plates and differentiated as aforementioned. Cells were incubated as aforementioned with MRI contrast agents diluted in phosphate-free media for 7 days. The oxygen consumption rate and extracellular acidification rate were measured using a Seahorse XFe96 Analyzer with the Cell Mito Stress Test Kit (Bucher 103015-100) and FluxPack (Bucher 102601-100) using BaseMedium (Bucher 102353-100) supplemented with D-glucose (Merck 1.08337.5000), sodium pyruvate (Sigma P5280), and L-glutamine (ThermoFisher 25030081), and adjusted to $\mathrm{pH} 7.4$ and sterile filtered according to the manufacturer's instructions. One micromolar of oligomycin A, $1.5 \mu \mathrm{M}$ of FCCP, and $0.5 \mu \mathrm{M}$ of rotenone/antimycin A were injected to assay the basal respiration rate, ATP production, maximal respiration, and nonmitochondrial oxygen consumption. Data were analyzed with Agilent Wave 2.4 software and macros, with cell numbers normalized by visual counting and CASY cell counts (OMNI Life Science) after each experiment.

\section{Statistics}

Two-tailed $t$-tests for independent means were used to compare each test sample to the negative control for statistical significance. Calculations were performed using the $t$-value calculator: https://www. socscistatistics.com/tests/studentttest/Default2.aspx.

\section{RESULTS}

\section{Differentiated Human Neurons Display Neural Morphology and Express Dopaminergic Markers}

Neurons were differentiated from a human neuroblastoma cell line into dopaminergic neurons as a representative model of some neurons in the basal ganglia, which is one of the 2 brain regions exhibiting highest T1 signal augmentation after repeat administration of GBCAs. ${ }^{32}$ Differentiation proceeded with retinoic acid followed by a phorbol ester and neural-specific growth factors according to the protocol in Figure 1, A and B. After 7 days of further culture in phosphate-free media without contrast agents, cells displayed an interconnected network of elongated axons (Fig. 1C), in contrast to the round cell morphology and short dendritic projections of the neuroblasts (Fig. 1A). mRNA expression analysis verified significantly increased expression of markers of mature dopaminergic neurons, including dopamine receptors, dopamine transporter, and mature neuron-specific enzymes, transcription factors, and structural proteins (Fig. 1D).

We next used differentiated human neurons to investigate cellular toxicity due to exposure to GBCAs. Each GBCA was applied at $50 \mu \mathrm{M}$, $100 \mu \mathrm{M}, 1 \mathrm{mM}$, and $10 \mathrm{mM}$ concentrations. A range of concentrations of gadolinium have been reported to have been measured in human brain tissue of autopsy patients who received various different GBCAs and numbers of doses, ${ }^{21,26,27}$ with higher concentrations reported by McDonald et al. With a molecular weight of $157.25 \mathrm{~g} / \mathrm{mol}$ for gadolinium, $20 \mu \mathrm{g} \mathrm{Gd} / \mathrm{g}$ brain tissue measured in some autopsy patients with repeat GBCA exposure ${ }^{21}$ corresponds to $127 \mu \mathrm{M}$ concentration of gadolinium in brain tissue, although this value is an approximation as the amount of water lost during tissue desiccation is uncertain. Fifty micromolar and $100 \mu \mathrm{M}$ were selected as "clinically relevant" concentrations after receipt of repeated doses of GBCA, and $1 \mathrm{mM}$ and $10 \mathrm{mM}$ were tested to reveal toxicity trends with higher dose.

\section{Cell Death Increases With Increasing GBCA Dose and Lower Kinetic Stability of the Agent}

For neurons exposed to GBCAs, the percentage of dead cells labeled by propidium iodide due to membrane compromise and apoptotic

TABLE 3. qRT-PCR Primers

\begin{tabular}{|c|c|c|}
\hline Target & Forward & Reverse \\
\hline Neurogenin 1 & 5'-AGCGCCTTTCTATCTGTCCG-3' & 5'-AGGAAGCCGGATAGGTCACT-3' \\
\hline Tau & 5'-ATGCACCAAGACCAAGAGGG-3' & 5'-CCGCTGTTGGAGTGCTCTTA-3' \\
\hline Dopamine receptor 2 & 5'-GGCTCCACTAAAGGAGGCTG-3' & 5'-GTTTTGCCATTGGGCATGGT-3' \\
\hline Dopamine receptor 3 & 5'-ACCCCACGAATGTTTCAGGA-3' & 5'-ATGCCAAGGGGCTTCACATT-3' \\
\hline Dopamine transporter & 5'-TCACCACCTCCATCAACTGC-3' & 5'-TGATGAAGAAGACCACCGCC-3' \\
\hline Neural enolase 2 & 5'-TGTGCATAGTGCTGGGATGG-3' & 5'-GACACATCGTTCCCCCAAGT-3' \\
\hline Nestin & 5'-GTAGCTCCCAGAGAGGGGAA-3' & 5'-CTCTAGAGGGCCAGGGACTT-3' \\
\hline Beta-actin & 5'-CGAGGACTTTGATTGCACATTGTT-3' & 5'-TGGGGTGGCTTTTAGGATGG-3' \\
\hline
\end{tabular}




\section{Culture, differentiation, characterization of human neurons}

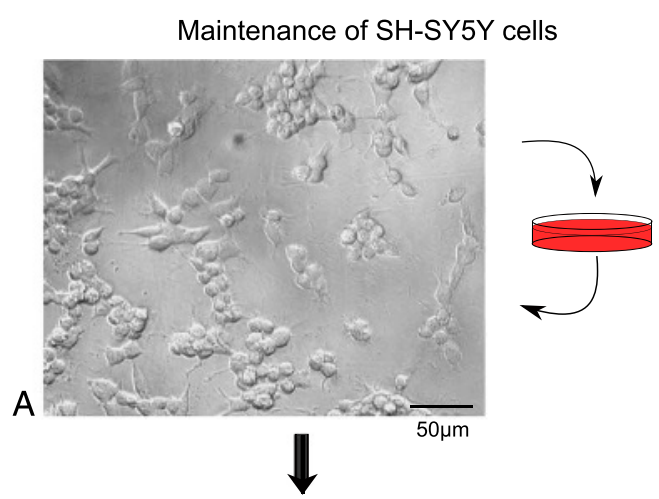

Thaw SH-SY5Y cells.

Culture in DMEM/F12

with stable L-glutamine,

$15 \%$ FBS, $1 \%$ NEAA,

$1 \% \mathrm{P} / \mathrm{S}$.

Change media every

3-4 days, also collecting

cells in suspension.

Passage $1: 10$ or higher when $80 \%$ confluent.

Differentiation into dopaminergic neurons

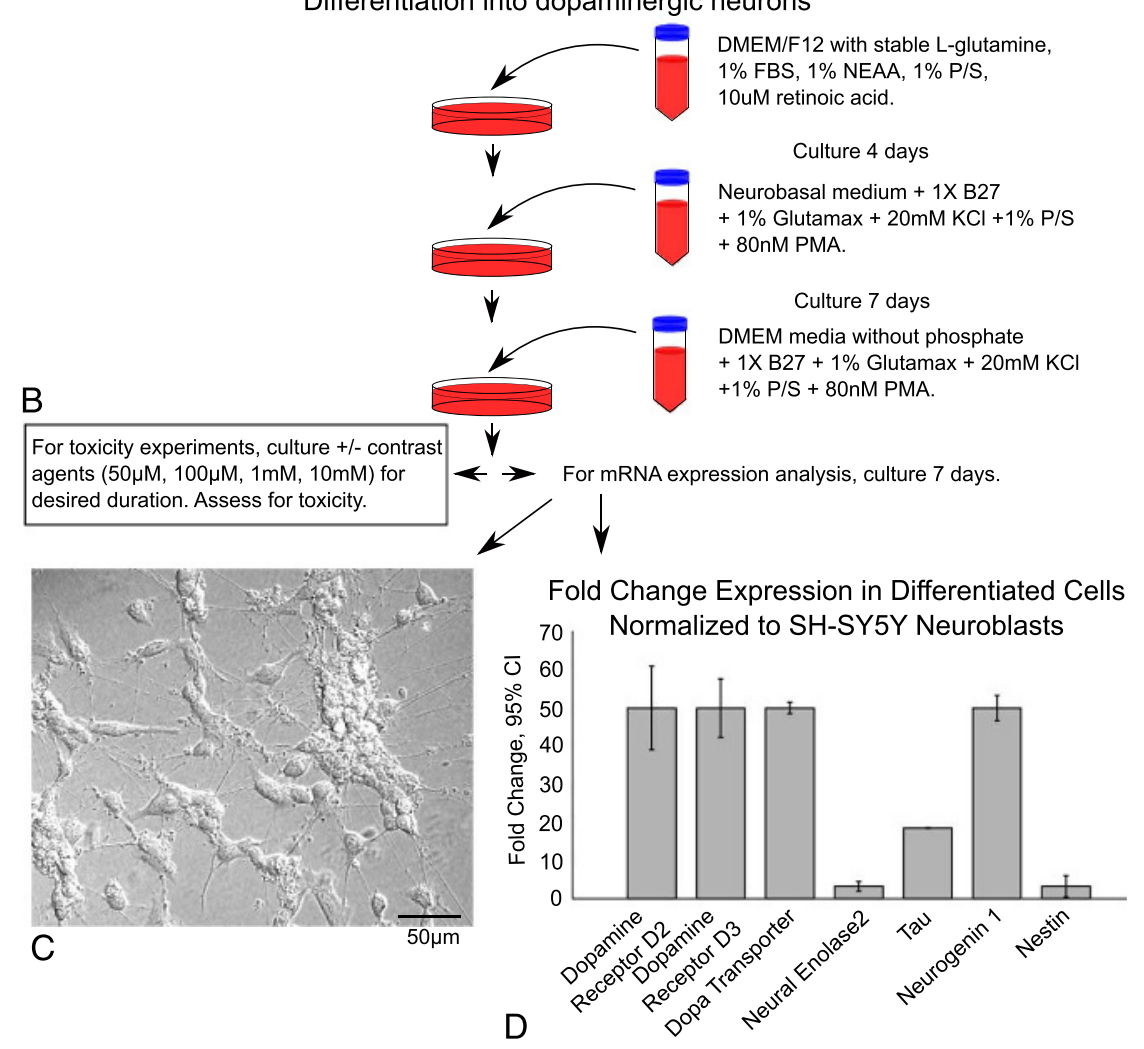

FIGURE 1. Differentiation of human neurons that highly express dopaminergic markers and display neural morphology. A, Undifferentiated human SH-SY5Y neuroblastoma cells are maintained in culture. Image depicts these nondifferentiated cells. B, Differentiation proceeds in 3 steps utilizing retinoic acid and a phorbol ester alongside depletion of serum and phosphate and provision of neural growth factors as shown. Differentiated neurons display elaborate axon networks (C) and highly express markers of dopaminergic neurons (D), fold change in expression and $95 \%$ confidence interval compared with undifferentiated cells. Fold change in expression greater than 50 was plotted as 50 .

cells labeled with caspase reagent were quantified relative to total cell number and normalized to negative controls that had not been exposed to contrast agents (Fig. 2). In general, caspase reagent labeled slightly higher proportions of cells than propidium iodide. This was likely due to apoptotic cells activating caspase before membrane integrity became compromised.

After 7 days of exposure, cells treated with nonformulated (NF) gadodiamide and gadodiamide showed the highest rates of cell death at 1.77- and 1.52-fold, respectively, relative to negative controls at $50 \mu \mathrm{M}$ (Fig. 2A). At $10 \mathrm{mM}$ of gadodiamide, dead cell numbers were double that of negative controls. Gadopentetate dimeglumine also caused statistically higher rates of cell death (approximately 1.25-fold) compared with controls at $50 \mu \mathrm{M}$ and $100 \mu \mathrm{M}$.
With gadoxetate disodium and gadobenate dimeglumine, cell death was only statistically elevated at $1 \mathrm{mM}$ and $10 \mathrm{mM}$ (to approximately 1.5 - to 1.65 -fold relative to controls).

The macrocyclic agents gadoterate meglumine, gadobutrol, and gadoteridol demonstrated no statistical increase in cell death at $50 \mu \mathrm{M}$ or $100 \mu \mathrm{M}$ (Fig. 2A). Cell death increased with dose to approximately 1.55-fold (gadoterate meglumine), 1.30-fold (gadobutrol), and 1.4-fold (gadoteridol) at $10 \mathrm{mM}$. The osmolality control mannitol caused no increased cell death compared with negative controls. Figure 2, B to D show selected representative images of the labeled cell nuclei and dead cell markers for gadopentetate dimeglumine (Fig. 2B), gadoteridol (Fig. 2C), and negative control (Fig. 2D). 


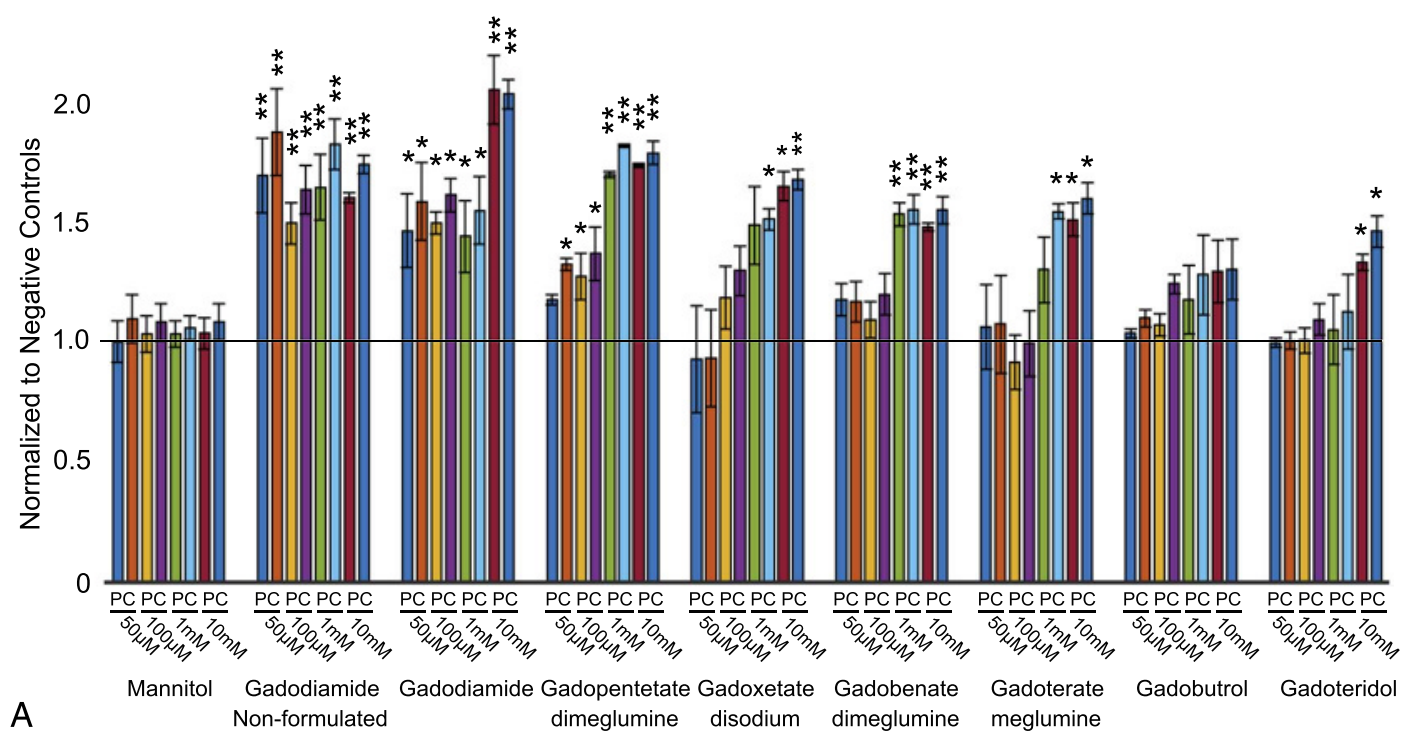

Gadopentetate dimeglumine
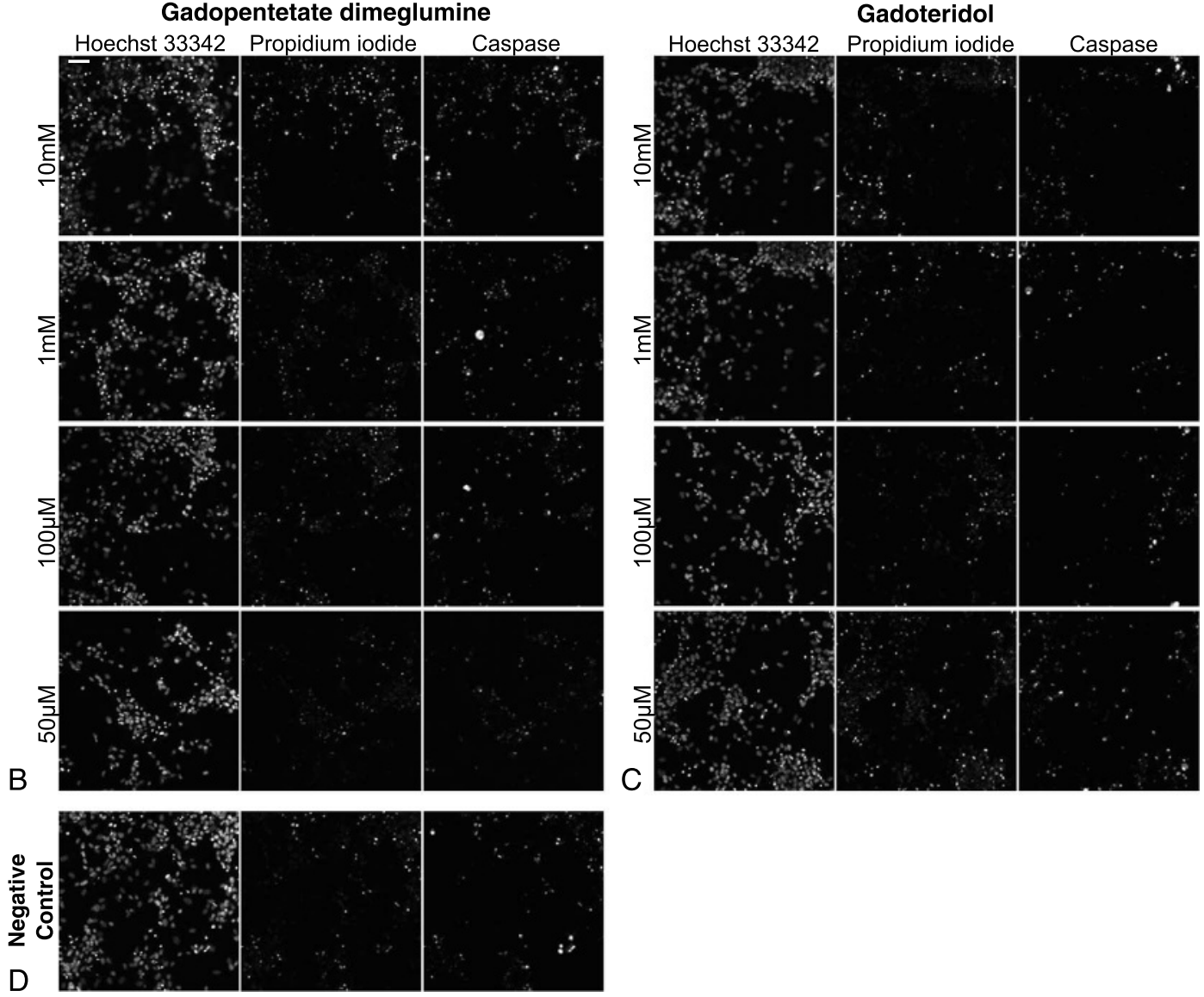

FIGURE 2. Cell viability drops with lower kinetic stability contrast agents and higher dose. Neurons incubated without (negative control) or with the specified agent and dose for 7 days were assayed for propidium iodide (P) uptake or positive caspase indicator (C) to quantify dead and apoptotic cells, respectively. A, The plot depicts the mean and SEM (error bars) of the proportion of dead and apoptotic cells normalized to negative controls at each tested concentration for the osmolality control mannitol and each contrast agent. $* P<0.05 ; * * P<0.01$. B to $D$, The images show representative examples of cells labeled for Hoechst 33342 (all cell nuclei), propidium iodide, or caspase at each concentration for samples treated with (B) gadopentetate dimeglumine, (C) gadoteridol, and (D) no agent (negative control). Scale bar = $50 \mu \mathrm{m}$ for all images. 


\section{Mitochondrial Membrane Potential Decreases With Increasing GBCA Dose and Lower Kinetic Stability of the Agent}

Next, we investigated a potential effect of GBCAs on mitochondrial function. A reduction in mitochondrial membrane potential impairs ATP production and can lead to apoptosis. ${ }^{33}$

After 7 days of incubation with GBCAs, mitochondrial membrane potential decreased with increasing concentration of contrast agent (Fig. 3). The potential also decreased more as the kinetic stability of the agents decreased. The most severe reduction in membrane potential was caused by NF-gadodiamide $(0.71$ relative to a control value of 1 at $50 \mu \mathrm{M}$ and 0.36 at $10 \mathrm{mM}$; Fig. 3A). Gadodiamide and gadopentetate dimeglumine caused comparable impairment (approximately 0.71 relative to controls at $50 \mu \mathrm{M}$ and 0.58 at $10 \mathrm{mM}$ ). Gadoxetate disodium caused less, but still statistically significant, impairments across the dose range. Gadobenate dimeglumine showed minimal effect at low dose but severe impairment at higher doses
( 0.70 and 0.50 relative to controls at $1 \mathrm{mM}$ and $10 \mathrm{mM}$, respectively; Fig. 3, A and B).

The macrocyclic agents generally exhibited less impact. After 7 days, membrane potential ranged from 0.96 (gadobutrol) to no impact (gadoterate meglumine and gadoteridol) at the lowest 2 concentrations (Fig. 3A).

At $10 \mathrm{mM}$, potential dropped to 0.75 (gadoterate meglumine; Fig. 3, A and C) and around 0.94 for gadobutrol and gadoteridol. The osmolality control mannitol caused no impact on membrane potential at any dose. Figure 3, B and C depict selected representative images of the cells.

\section{Mitochondrial Oxidative Respiratory Capacity Decreases With Increasing GBCA Dose and Lower Kinetic Stability of the Agent}

Because mitochondrial membrane potential was affected by GBCAs, we tested for a corresponding impairment in mitochondrial oxidative respiratory function (Fig. 4). The fluorescent indicator
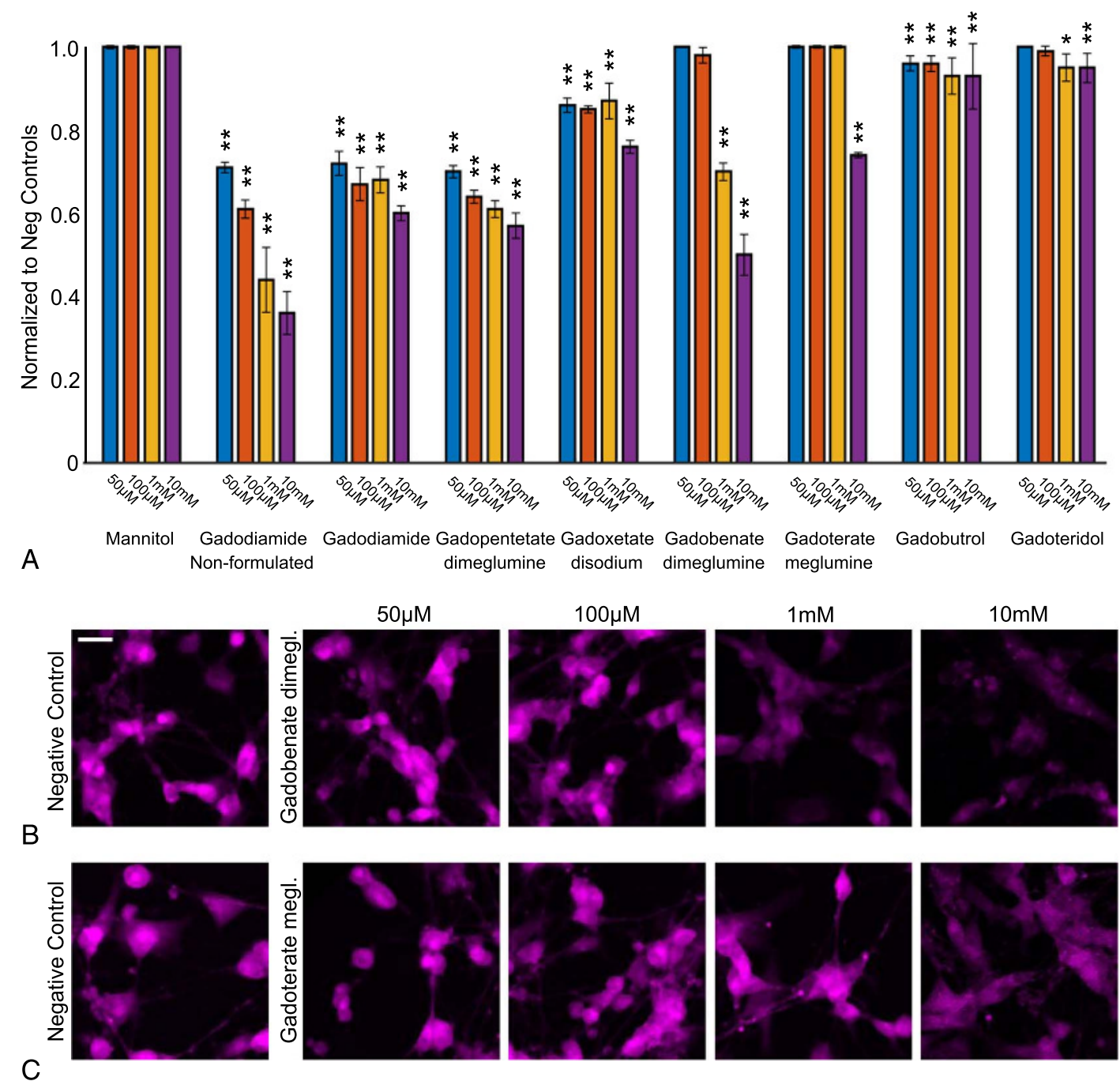

FIGURE 3. Mitochondrial membrane potential weakens with lower kinetic stability contrast agents and higher dose. The mitochondrial membrane potential was quantified in neurons incubated without (negative control) or with the specified agent and dose for 7 days. A, The graph depicts the mean and SEM (error bars) of the membrane potential of neurons treated with each agent, normalized to negative controls. $* P<0.05 ; * * P<0.01$. $B$ and $C$, The images depict the fluorescent mitochondrial membrane potential indicator in representative examples of neurons treated with (B) gadobenate dimeglumine or (C) gadoterate meglumine in comparison to neurons treated without agent (negative controls). Scale bar $=25 \mu \mathrm{m}$ for all images. 

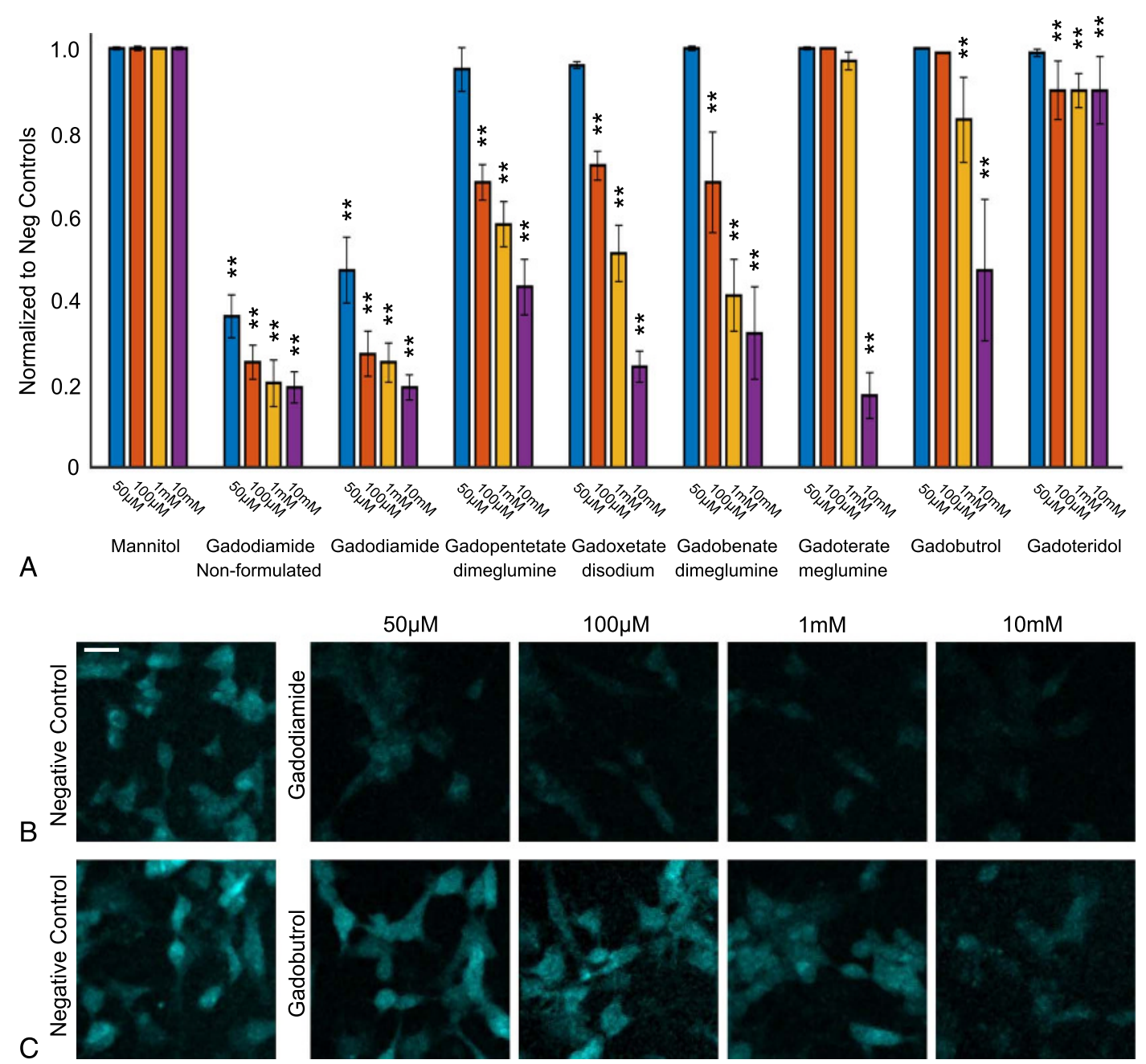

FIGURE 4. Mitochondrial oxidative respiratory capacity diminishes with lower kinetic stability contrast agents and higher dose. The mitochondrial oxidative respiratory capacity was quantified in neurons treated without (negative control) or with the specified agent and dose for 7 days. A, The plot depicts the mean and SEM (error bars) of the oxidative capacity of neurons treated with each agent, normalized to negative controls. $* P<0.05$; $* * P<0.01$. B and C, The images depict the fluorescent indicator in representative examples of neurons treated with (B) gadodiamide or (C) gadobutrol in comparison to neurons treated without agent (negative controls). Scale bar $=25 \mu \mathrm{m}$ for all images.

DCFH2-DA enters the mitochondrial matrix where it is oxidized to a fluorescent state by mitochondrial reactive oxygen species from oxidative phosphorylation and thereby serves as an effective indicator of mitochondrial oxidative respiratory function. ${ }^{34}$

For all of the contrast agents, mitochondrial oxidative function decreased with higher agent concentration. After 7 days of incubation with GBCAs, gadodiamide and NF-gadodiamide again exhibited the greatest impairment, with mitochondrial oxidative capacity dropping to 0.47 (gadodiamide) or 0.36 (NF-gadodiamide) compared with the capacity of negative controls with $50 \mu \mathrm{M}$ agent concentration (Fig. 4, A and B). At $10 \mathrm{mM}$ concentration of gadodiamide and NF-gadodiamide, the mitochondrial oxidative capacity was reduced to 0.19 compared with the capacity of negative controls. The other linear agents had no statistical impact at $50 \mu \mathrm{M}$, but oxidative function dropped to 0.43 (gadopentetate dimeglumine), 0.24 (gadoxetate disodium), and 0.32 (gadobenate dimeglumine) the capacity of negative controls with $10 \mathrm{mM}$ agent concentration.

The macrocyclic agents showed no impairment at $50 \mu \mathrm{M}$ and much less impairment than the linear agents up to $1 \mathrm{mM}$ concentration (Fig. 4, A-C). At $10 \mathrm{mM}$, oxidative capacity was 0.17 (gadoterate meglumine), 0.47 (gadobutrol), and 0.90 (gadoteridol) compared with that of controls. The osmolality control mannitol demonstrated no effect on mitochondrial oxidative function for any concentration tested. Figure 4, B and C show selected representative images of the cells.

\section{Mitochondrial Function Measured by Oxygen Consumption Decreases With Increasing GBCA Dose and Lower Kinetic Stability of the Agent}

The mitochondrial stress test implemented with the Seahorse XFe96 Analyzer further assesses mitochondrial function by measuring the oxygen consumption rate and extracellular acidification rate of living cells, from which various respiratory parameters and ATP production can be measured (Fig. 5). After 7 days of incubation with $50 \mu \mathrm{M}$ or $100 \mu \mathrm{M}$ GBCA, no statistically significant differences in basal respiration rate, ATP production, maximal respiration, or spare respiratory capacity (maximal-basal respiration) were measured (Fig. 5, A and B). At $1 \mathrm{mM} \mathrm{GBCA}$ concentration, statistically significant impairments in maximal respiration and spare capacity were observed for NF-gadodiamide (Fig. 5C). 


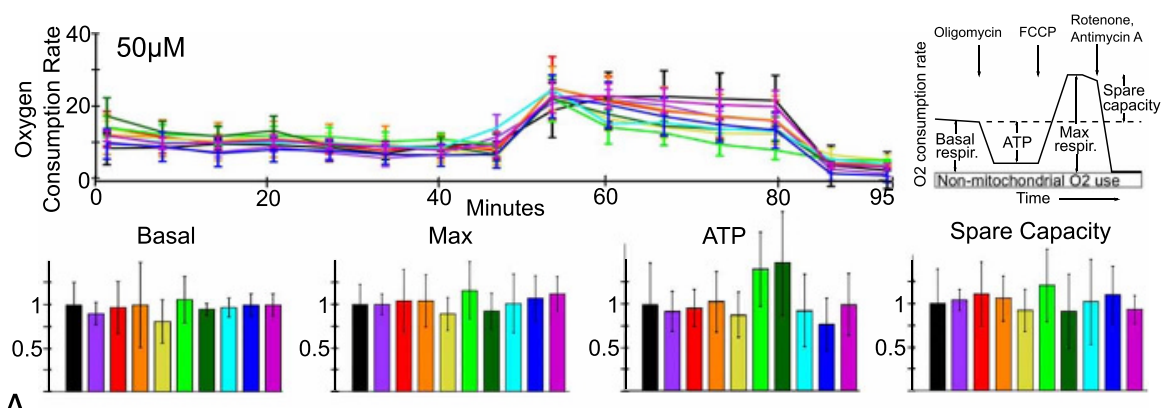

A
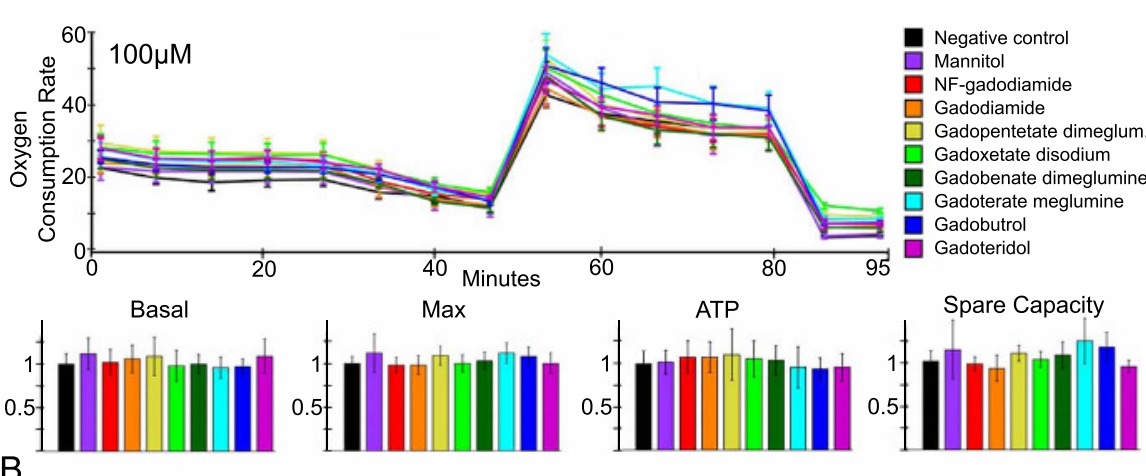

B
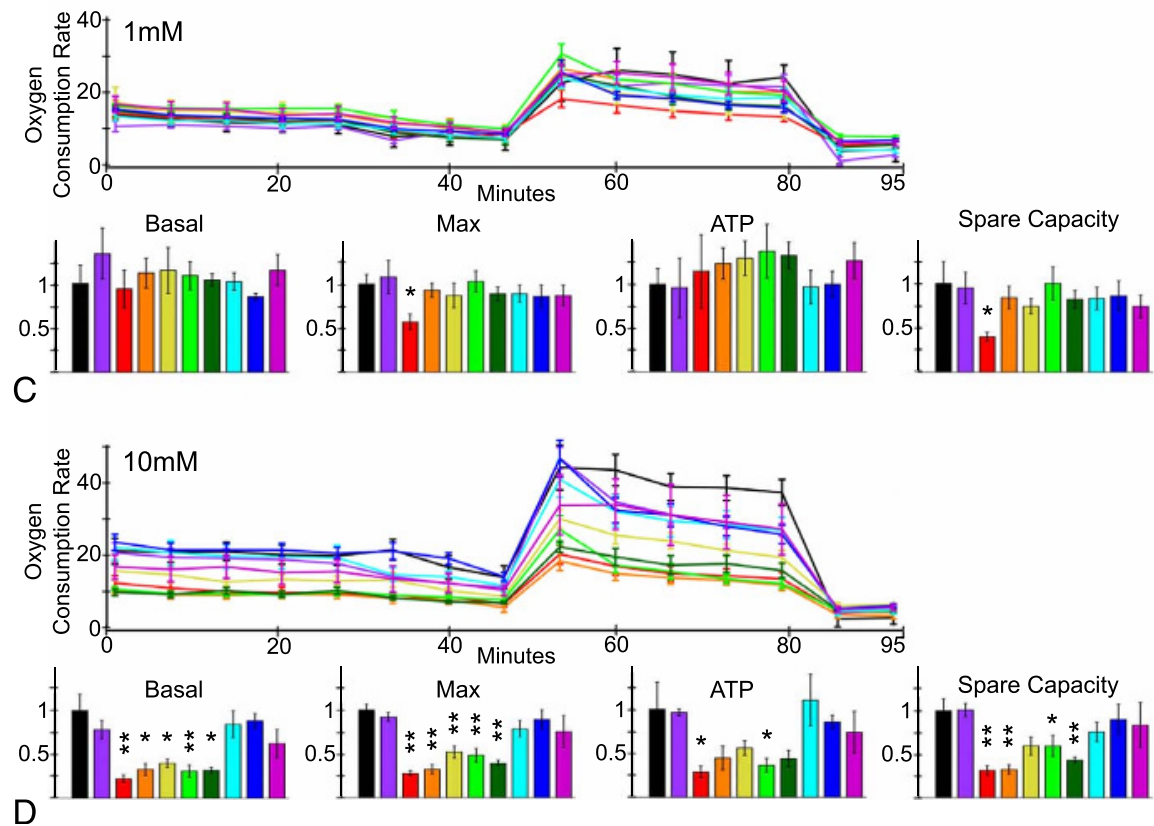

FIGURE 5. Mitochondrial function measured by oxygen consumption declines with lower kinetic stability contrast agents and higher dose. Oxygen consumption and extracellular acidification rates were measured with a Seahorse XFe96 Analyzer. Basal and maximal respiratory rates, ATP production, and spare respiratory capacity were quantified. Line graphs plot the oxygen consumption rate (pmol/min/cell count). Bar plots depict the mean and SEM (error bars) of the derived respiratory parameters for neurons incubated for 7 days without (negative control) or with each specified agent at concentrations of (A) $50 \mu \mathrm{M},(B) 100 \mu \mathrm{M},(C) 1 \mathrm{mM}$, and (D) $10 \mathrm{mM}$. $* P \leq 0.05$; ** $P<0.01$.

With 10 mM GBCA concentration, the linear agents demonstrated substantially reduced basal and maximal respiration, spare capacity, and ATP production (Fig. 5D). The spare capacity for gadopentetate dimeglumine and ATP production for gadodiamide, gadopentetate dimeglumine, and gadobenate dimeglumine fell short of statistical significance $(P=0.08, P=0.12, P=0.23$, $P=0.10$, respectively), although the standard errors do not overlap with that of the negative control.
With $10 \mathrm{mM}$ GBCA concentration, the macrocyclic agents demonstrated some, but less, impairment in all measures, and none achieved statistically significant deviation from controls (Fig. 5D).

\section{DISCUSSION}

The growing evidence that GBCAs remain in brain tissue on the order of months or longer after administration, and that the less 
kinetically stable agents dissociate, producing soluble or insoluble gadolinium species that may persist for an indefinite period, necessitates understanding the potential impacts of these agents on cellular function. In the current study, we demonstrate that GBCAs induce cell death and have toxic effects on the mitochondrial function of differentiated human neurons.

Fifty micromolar and $100 \mu \mathrm{M}$ concentrations of the contrast agents were tested as clinically relevant doses because these concentrations correspond to reported amounts of gadolinium measured in brain tissue of some patients at autopsy after repeated contrast-enhanced MRI scans. ${ }^{21}$ Higher concentrations of $1 \mathrm{mM}$ and $10 \mathrm{mM}$ were also tested to reveal dose-response toxicity trends.

The highest rates of cell death were caused by formulated and NF-gadodiamide, followed successively by gadopentetate dimeglumine, gadoxetate disodium, and gadobenate dimeglumine. The macrocyclics induced less cell death than the linear contrast agents. Except for the NF-gadodiamide, which showed high rates of cell death across all concentrations, for all other agents, cell death broadly increased with agent concentration. At concentrations of $50 \mu \mathrm{M}$ and $100 \mu \mathrm{M}$, formulated and NF-gadodiamide, and gadopentetate dimeglumine statistically significantly increased cell death compared with negative controls.

Mitochondrial membrane potential and mitochondrial oxidative capacity decreased with increasing concentration for all agents. Nonformulated gadodiamide caused the greatest impairments, followed by the other linear agents. All linear agents caused statistically significant reduction of mitochondrial membrane potential and oxidative capacity at $50 \mu \mathrm{M}$ and $100 \mu \mathrm{M}$ concentrations, except that the potential reduction for gadobenate dimeglumine did not reach statistical significance until higher concentrations were applied. Macrocyclic agents caused less effect, particularly at $50 \mu \mathrm{M}$ and $100 \mu \mathrm{M}$ concentrations, and statistically significant impairments at these concentrations (membrane potential with gadobutrol and oxidation with gadoteridol) were lower in magnitude. Likewise, NF-gadodiamide was first to elicit impairments in mitochondrial function measured by oxygen consumption, and at the highest concentration tested, all linear agents but no macrocyclics induced statistically significant functional impairments. The osmolality control mannitol demonstrated no increased cell death or impact on mitochondrial function in any of the assays used, at any of the concentrations tested.

Across 5 independent measures of toxicity that we tested, our results consistently demonstrate that cellular toxicity increases with dose for all contrast agents, and toxicity broadly increases with decreasing kinetic stability of the contrast agent. These findings are consistent with the association between lower kinetic stability agents having greater incidence of toxicity in other tissues, for instance nephrogenic systemic fibrosis arising in patients with compromised renal function. ${ }^{35,36}$ The mitochondrial toxicity may lead directly to caspase-induced cell death via well-described pathways, such as involving cytochrome $\mathrm{C} .{ }^{37} \mathrm{In}-$ deed, in rat liver mitochondria, gadolinium causes mitochondrial swelling by opening the mitochondrial permeability transition pore, dissipates the inner membrane potential, alters mitochondrial membrane fluidity, and induces apoptosis via cytochrome $\mathrm{C}$ release. ${ }^{38}$ Our results are also consistent with a study performed in rat neurons, which found that, within 24 hours, gadolinium chloride induces mitochondrial dysfunction and cell death. ${ }^{39}$ Other cell types exhibit similar mitochondrial toxicity in response to gadolinium. Treatment of various multiple myeloma cell lines with motexafin gadolinium in the presence of ascorbate increases reactive oxygen species production, decreases mitochondrial membrane potential, and induces apoptosis via induction of annexin $\mathrm{V}^{40}$ Likewise, exposing murine macrophages to GBCAs at concentrations as low as $2.5 \mu \mathrm{M}$ induces mitochondrial stress with reactive oxygen species production and reduced mitochondrial membrane potential and alters inflammatory cytokine production. ${ }^{41}$ This study also found greater impairments after treatment with linear agents in comparison to the macrocyclic gadobutrol.
We assayed for cellular toxicity 1 week after administration of GBCAs. This is a long enough time-frame for cells to recover from immediate, transient toxic effects of the agents, and this indicates that cellular toxicity persists beyond the immediate time of administration. To exert an effect on mitochondrial function, the agents must be internalized within the cells, which is consistent with prior studies. ${ }^{42}$ We did not assay how much agent entered the cells in our system or the form of the gadolinium, that is, complex, dissociated ion, or bound to a macromolecule. It is possible that the extent of cellular uptake of an individual agent is the determining factor of the level of resulting toxicity. If dissociation and indeed binding to a macromolecule promote cellular uptake, then greater uptake of less kinetically stable agents could explain the observed results even if all agents, even in complexed form, are toxic once within a cell.

Robert et $\mathrm{al}^{17}$ demonstrated stable persistence up to 1 year of gadolinium bound to macromolecules in brains of rats treated with gadodiamide, in comparison to gadoterate being undetectable after 5 months, with no binding to macromolecules. Thus, in the brain, the duration of exposure of individual neurons to contrast agent is much longer than the 7 days of exposure that we tested. Integrated over extended periods, particularly with dissociation and persistence of the agents with lower kinetic stability, the effective concentration to which individual neurons are exposed could be considerably higher than for just 7 days of exposure at a given dose. Therefore, our results emphasize the importance of the complete clearance of intact complex from the brain (and other tissues), as persistence prolongs the opportunity for gadolinium to dissociate, particularly from the lower kinetic stability agents, and for gadolinium species to be taken up into cells. Similarly, the cumulative administered dose of gadolinium within a certain time frame is also important, as the total concentration will accrue if contrast-enhanced MRI scans are repeated before the contrast agent has completely cleared from the previous scan. Once they enter cells, our results indicate that these agents do cause toxicity and induce apoptosis in human neurons.

This study was conducted in cell culture, which has its inherent limitations in directly applying results to human beings. First, contrast agents in culture medium have easy access to cell membranes. Second, the medium itself is clearly different from the interstitium in human brain. Third, cells in culture inherently have a different environment to those in the brain and do not replicate the tissue and synaptic complexity in vivo. Nevertheless, controlled, systematic testing to identify and characterize a potential toxic effect of GBCAs necessitated an accessible, reproducible system, which cells in culture provide. We therefore were able to test all the trademark GBCAs that we could obtain.

In addition, a miscalculation in preparing our osmolality controls resulted in the osmolality of our highest concentration of mannitol not equaling the osmolalities of the highest osmolality contrast agents at the $10 \mathrm{mM}$ concentration: the highest osmolality of mannitol tested was $24 \mathrm{mOsm} / \mathrm{kg}$, compared with $27 \mathrm{mOsm} / \mathrm{kg}$ for gadoterate meglumine, $27.5 \mathrm{mOsm} / \mathrm{kg}$ for gadoxetate disodium, $39.2 \mathrm{mOsm} / \mathrm{kg}$ for gadopentetate dimeglumine, and $39.4 \mathrm{mOsm} / \mathrm{kg}$ for gadobenate dimeglumine. Thus, through the $1 \mathrm{mM}$ concentration, the osmolalities of all contrast agents were properly controlled, but for the highest concentration tested $(10 \mathrm{mM})$, the osmolalities of these 4 contrast agents was not fully controlled. Nevertheless, the measured toxicity did not parallel the osmolality of the agents. If osmolality had been responsible for the observed toxicity, we would have expected gadopentetate dimeglumine and gadobenate dimeglumine to be the most toxic, and this was not the case.

Finally, the ideal system for these experiments would have been to use primary human cerebellar neurons from the dentate nucleus; however, the difficulty of obtaining and culturing such neurons would greatly reduce the feasibility of conducting such a study. Utilizing differentiated human neurons from an established cell line ensured the feasibility and timeliness of the study. Within the basal ganglia, the globus 
pallidus receives afferent inputs from dopaminergic neurons from the substantia nigra, thus making these neurons relevant to the study of the basal ganglia.

Across all of the contrast agents and 5 different assessments of toxicity, we measured dose-dependent toxicity that reflected the kinetic stability of the agents. The consistency of these findings lends credibility to the result that GBCAs induce mitochondrial toxicity and death in human neurons. We also used differentiated human neurons of a class found within one of the most affected regions of the brain, which offers a closer replica of the human brain than using rodent neurons. Therefore, it is not unreasonable to project that neurons in the human brain would experience some degree of the toxicity observed in cultured human neurons.

These findings raise the important question of how clinically significant the observed toxicity is for a patient obtaining contrastenhanced MRI. At 50 and $100 \mu \mathrm{M}$ concentrations of GBCA, which are within the concentration range measured in brain tissue of some autopsy patients ${ }^{21}$ after repeated contrast-enhanced MRI scans, the lower stability agents consistently demonstrated toxic effects on mitochondrial function and cell viability. In the couple of instances where toxicity from macrocyclic agents was statistically significant at these doses, the magnitude of the effect was less. A single dose of GBCA for a single contrast-enhanced MRI scan would not be expected to achieve this concentration of agent within the brain. However, with numerous repeated administrations, and evidence that the lower stability agents may accumulate and persist longer, ${ }^{17,18}$ our findings suggest that repeated administration, particularly of the lower kinetic stability GBCAs, could result in neural toxicity.

Whether the cellular dysfunction could produce clinical effects remains an unanswered question. Nevertheless, loss of any neurons due to induced cell death is concerning, and any cellular toxicity may accelerate cell death due to normal aging, which could become clinically significant in older patients who have received multiple contrastenhanced MRIs over the course of their life. Likewise, extra caution in utilizing these agents should be taken in the background of neural disease, for instance Friedreich's ataxia, where toxicity to neurons such as in the cerebellar dentate nucleus, which is important for initiation and control of movement, could exacerbate the disease. ${ }^{43}$ As another example, patients with multiple sclerosis receive repeated contrast-enhanced MRI scans to monitor the course of their disease. A recent study found a correlation between higher T1 signal intensity in the dentate nucleus and lower verbal fluency after correcting for disease severity. ${ }^{44}$ Although the study does not prove causation, in light of our results that GBCAs do cause neural toxicity, it underscores the importance of contrast agent selection, particularly in the background of neural disease. More broadly, our findings support the growing body of evidence that, when contrast enhancement is clinically necessary, utilizing the lowest dose possible and contrast agents with higher kinetic stability and a lower toxicity profile is prudent.

Future studies are needed to assess toxicity more than 1 week after administration to clarify how long toxic effects persist after a single administration of GBCA. Furthermore, effects on other aspects of cellular function remain to be tested. Likewise, the magnitude of effects on cellular function of repeated administration of agents should ideally be tested in human neural tissue. However, the complexity of such experiments and difficulty in maintaining human brain tissue explants in culture may make rodents better suited to such studies. It will also be important to continue to monitor for clinical signs of neurological impairment in patients receiving repeated administration of GBCAs over their lifetime.

After much uncertainty as to whether GBCAs cause cellular toxicity, we now provide the first definitive evidence that these agents do indeed induce mitochondrial toxicity and cell death in cultured human neurons. The magnitude of the measured toxicity broadly increases as the kinetic stability of the contrast agent decreases, and the lower stability agents induce toxicity at concentrations that fall within the range detected in some autopsy patients. For all agents, the magnitude of the toxicity increases with concentration. These results shed light on a fundamental, long-standing question and highlight the importance of a more complete understanding of the safety of these agents at the cellular level.

\section{ACKNOWLEDGMENTS}

The authors thank all the members of the radiology laboratory group for the helpful discussions regarding this work, and to Stefan Freigang and his laboratory members for the assistance in utilizing the Seahorse XFe96 Analyzer.

\section{REFERENCES}

1. Caravan P, Ellison JJ, McMurry TJ, et al. Gadolinium(III) chelates as MRI contrast agents: structure, dynamics, and applications. Chem Rev. 1999;99:2293-2352.

2. Kanda T, Ishii K, Kawaguchi H, et al. High signal intensity in the dentate nucleus and globus pallidus on unenhanced T1-weighted MR images: relationship with increasing cumulative dose of a gadolinium-based contrast material. Radiology. 2014;270:834-841.

3. Errante Y, Cirimele V, Mallio CA, et al. Progressive increase of T1 signal intensity of the dentate nucleus on unenhanced magnetic resonance images is associated with cumulative doses of intravenously administered gadodiamide in patients with normal renal function, suggesting dechelation. Invest Radiol. 2014;49:685-690.

4. Radbruch A, Weberling LD, Kieslich PJ, et al. Gadolinium retention in the dentate nucleus and globus pallidus is dependent on the class of contrast agent. Radiology. 2015;275:783-791

5. Kanda T, Osawa M, Oba H, et al. High signal intensity in dentate nucleus on unenhanced T1-weighted MR images: association with linear versus macrocyclic gadolinium chelate administration. Radiology. 2015;275:803-809.

6. Bjørnerud A, Vatnehol SAS, Larsson C, et al. Signal enhancement of the dentate nucleus at unenhanced MR imaging after very high cumulative doses of the macrocyclic gadolinium-based contrast agent gadobutrol: an observational study. Radiology. 2017;285:434-444

7. Bolles GM, Yazdani M, Stalcup ST, et al. Development of high signal intensity within the globus pallidus and dentate nucleus following multiple administration of gadobenate dimeglumine. AJNR Am J Neuroradiol. 2018;39:415-420.

8. Weberling LD, Kieslich PJ, Kickingereder P, et al. Increased signal intensity in the dentate nucleus on unenhanced T1-weighted images after gadobenate dimeglumine administration. Invest Radiol. 2015;50:743-748.

9. Radbruch A, Weberling LD, Kieslich PJ, et al. High-signal intensity in the dentate nucleus and globus pallidus on unenhanced T1-weighted images: evaluation of the macrocyclic gadolinium-based contrast agent gadobutrol. Invest Radiol. 2015;50:805-810.

10. Radbruch A, Weberling LD, Kieslich PJ, et al. Intraindividual analysis of signal intensity changes in the dentate nucleus after consecutive serial applications of linear and macrocyclic gadolinium-based contrast agents. Invest Radiol. 2016; 51:683-690.

11. Can Y, Huang DQ, Shih G, et al. Signal change in the dentate nucleus on T1weighted MR images after multiple administrations of gadopentetate dimeglumine versus gadobutrol. AJR Am J Roentgenol. 2016;206:414 419.

12. Radbruch A, Haase R, Kieslich PJ, et al. No signal intensity increase in the dentate nucleus on unenhanced T1-weighted MR images after more than 20 serial injections of macrocyclic gadolinium-based contrast agents. Radiology. 2017;282 699-707

13. Iliff JJ, Wang M, Liao Y, et al. A paravascular pathway facilitates CSF flow through the brain parenchyma and the clearance of interstitial solutes, including amyloid B. Sci Transl Med. 2012;4:147ra111.

14. Nedergaard M, Goldman SA. Brain drain. Sci Am. 2016;314:44-49.

15. Tarasoff-Conway JM, Carare RO, Osorio RS, et al. Clearance systems in the brainimplications for Alzheimer disease. Nat Rev Neurol. 2015;11:457-470.

16. Taoka T, Jost G, Frenzel T, et al. Impact of the glymphatic system on the kinetic and distribution of gadodiamide in the rat brain: observations by dynamic MR and effect of circadian rhythm on tissue gadolinium concentrations. Invest Radiol. 2018;53:529-534.

17. Robert P, Fingerhut S, Factor C, et al. One-year retention of gadolinium in the brain: comparison of gadodiamide and gadoterate meglumine in a rodent model. Radiology. 2018;288:424-433.

18. Frenzel T, Apte C, Jost G, et al. Quantification and assessment of the chemical form of residual gadolinium in the brain after repeated administration of gadolinium-based contrast agents: comparative study in rats. Invest Radiol. 2017;52:396-404 
19. Gianolio E, Bardini P, Arena F, et al. Gadolinium retention in the rat brain: assessment of the amounts of insoluble gadolinium-containing species and intact gadolinium complexes after repeated administration of gadolinium-based contrast agents. Radiology. 2017;285:839-849.

20. Rasschaert M, Schroeder JA, Wu TD, et al. Multimodal imaging study of gadolinium presence in rat cerebellum: differences between Gd chelates, presence in the Virchow-Robin space, association with lipofuscin, and hypotheses about distribution pathway. Invest Radiol. 2018;53:518-528.

21. McDonald RJ, McDonald JS, Kallmes DF, et al. Gadolinium deposition in human brain tissues after contrast-enhanced MR imaging in adult patients without intracranial abnormalities. Radiology. 2017;285:546-554.

22. Burke LM, Ramalho M, AlObaidy M, et al. Self-reported gadolinium toxicity: a survey of patients with chronic symptoms. Magn Reson Imaging. 2016;34: 1078-1080.

23. Semelka RC, Ramalho J, Vakharia A, et al. Gadolinium deposition disease: initial description of a disease that has been around for a while. Magn Reson Imaging. 2016;34:1383-1390.

24. Semelka RC, Commander CW, Jay M, et al. Presumed gadolinium toxicity in subjects with normal renal function: a report of 4 cases. Invest Radiol. 2016;51: 661-665.

25. Ramalho M, Ramalho J, Burke LM, et al. Gadolinium retention and toxicity-an update. Adv Chronic Kidney Dis. 2017;24:138-146.

26. Kanda T, Fukusato T, Matsuda M, et al. Gadolinium-based contrast agent accumulates in the brain even in subjects without severe renal dysfunction: evaluation of autopsy brain specimens with inductively coupled plasma mass spectroscopy. Radiology. 2015;276:228-232.

27. Murata N, Gonzalez-Cuyar LF, Murata K, et al. Macrocyclic and other non-group 1 gadolinium contrast agents deposit low levels of gadolinium in brain and bone tissue: preliminary results from 9 patents with normal renal function. Invest Radiol. 2016;51:447-453.

28. Idée JM, Fretellier N, Robic C, et al. The role of gadolinium chelates in the mechanism of nephrogenic systemic fibrosis: a critical update. Crit Rev Toxicol. 2014; 44:895-913.

29. Port M, Idée JM, Medina C, et al. Efficiency, thermodynamic and kinetic stability of marketed gadolinium chelates and their possible clinical consequences: a critical review. Biometals. 2008;21:469-490.

30. Runge VM, Ai T, Hao D, et al. The developmental history of the gadolinium chelates as intravenous contrast media for magnetic resonance. Invest Radiol. 2011; 46:807-816.
31. Crowley LC, Marfell BJ, Waterhouse NJ. Analyzing cell death by nuclear staining with Hoechst 33342. Cold Spring Harb Protoc. 2016;2016.

32. Zhang Y, Cao Y, Shih GL, et al. Extent of signal hyperintensity on unenhanced T1-weighted brain MR images after more than 35 administrations of linear gadolinium-based contrast agents. Radiology. 2017;282:516-525.

33. Li PF, Dietz R, von Harsdorf R. p53 regulates mitochondrial membrane potential through reactive oxygen species and induces cytochrome $c$-independent apoptosis blocked by Bcl-2. EMBO J. 1999;18:6027-6036.

34. Akopova OV, Kolchinskaya L, Nosar V. The use of dichlorofluorescein as a probe for monitoring the effects of calcium on ROS production in mitochondria. Biochem Anal Biochem. 2017;6:1000334.

35. Sieber MA, Lengsfeld P, Frenzel T, et al. Preclinical investigation to compare different gadolinium-based contrast agents regarding their propensity to release gadolinium in vivo and to trigger nephrogenic systemic fibrosis-like lesions. Eur Radiol. 2008;18:2164-2173.

36. Thomsen HS, Morcos SK, Dawson P. Is there a causal relation between the administration of gadolinium based contrast media and the development of nephrogenic systemic fibrosis (NSF)? Clin Radiol. 2006;61:905-906.

37. Green DR, Reed JC. Mitochondria and apoptosis. Science. 1998;281:1309-1312.

38. Zhao J, Zhou ZQ, Jin JC, et al. Mitochondrial dysfunction induced by different concentrations of gadolinium ion. Chemosphere. 2014;100:194-199.

39. Feng X, Xia Q, Yuan L, et al. Impaired mitochondrial function and oxidative stress in rat cortical neurons: implications for gadolinium-induced neurotoxicity. Neurotoxicology. 2010;31:391-398.

40. Evens AM, Lecane P, Magda D, et al. Motexafin gadolinium generates reactive oxygen species and induces apoptosis in sensitive and highly resistant multiple myeloma cells. Blood. 2005; 105:1265-1273.

41. Weng TI, Chen HJ, Lu CW, et al. Exposure of macrophages to low-dose gadolinium-based contrast medium: impact on oxidative stress and cytokines production. Contrast Media Mol Imaging. 2018;2018:3535769.

42. Cabella C, Crich SG, Corpillo D, et al. Cellular labeling with Gd(III) chelates only high thermodynamic stabilities prevent the cells acting as 'sponges' of $\mathrm{Gd} 3$ + ions. Contrast Med Mol Imaging. 2006;1:23-29.

43. Lodi R, Cooper JM, Bradley JL, et al. Deficit of in vivo mitochondrial ATP production in patients with Friedreich ataxia. Proc Natl Acad Sci U S A. 1999;96: 11492-11495.

44. Forslin Y, Shams S, Hashim F, et al. Retention of gadolinium-based contrast agents in multiple sclerosis: retrospective analysis of an 18-year longitudinal study. AJNR Am J Neuroradiol. 2017;38:1311-1316. 